Highly Energetic Physical Processes and

Mechanisms for Emission from Astrophysical Plasmas

IAU Symposium, Vol. 195, 2000

P. C. H. Martens, S. Tsuruta, and M. A. Weber, eds.

\title{
A Scaling Law for Magnetic Flux Tubes on an AGN Accretion Disk
}

\author{
A. M. K. LeRoux, D. W. Longcope, and S. Tsuruta \\ Physics Department, Montana State University, Bozeman, MT 59717, \\ U.S.A.
}

\begin{abstract}
An ASCA observation of Seyfert galaxy NGC 3227 showed flares with a linear increase and exponential decrease similar to that of solar flares. We derive a scaling law relating the loop length of a magnetic flux tube to rise and decay times of the flare using cooling mechanisms suitable for the central engine of a Seyfert galaxy. The predicted loop lengths are consistent with physical constraints on the plasma dynamics, suggesting that the same mechanism which explains solar flares may explain variability in Seyfert galaxies.
\end{abstract}

\section{Introduction}

Magnetic reconnection is the hypothesized cause of solar flares (Lin \& Hudson 1976) and flares in X-ray stars (Hawley et al. 1995). In keeping with early work suggesting an AGN corona may consist of many magnetic flux tubes filled with hot plasma emerging from a relatively cold, dense accretion disk (Rosner, Tucker, \& Vaiana 1977) and more recent work describing the structure of such flux tubes (DiMatteo 1998), we derive a scaling law for AGN flares.

\section{Loop Scaling Law for an AGN}

A recent ASCA observation of NGC 3227 showed flares with linear increase and exponential decay similar to those characteristic of solar flares (LeRoux et al., in preparation). While solar flare dynamics are dominated by thermal bremsstrahlung, AGN accretion disk cooling mechanisms also include synchrotron and inverse Compton cooling. Following the method of Fisher \& Hawley (1990), a new scaling law is derived which includes the effects of the additional cooling mechanisms and relates loop length $L$ to the apex temperature $T_{A}$, flare rise time $\tau_{R}$, and flare decay time $\tau_{D}$, and is of form:

$$
L=a T_{A}^{s} \tau_{R}^{p} \tau_{D}^{q}
$$

where $a, s, p$, and $q$ are coefficients determined by the temperature regime of thermal bremsstrahlung.

The resulting loop lengths as a function of apex temperature are shown in Figure 1 for each of 5 flares measured on NGC 3227. The loop must be short enough that sound can cross it during the flare, have thermal energy consistent with the energy emitted during the flare, and be longer than the pressure scale 


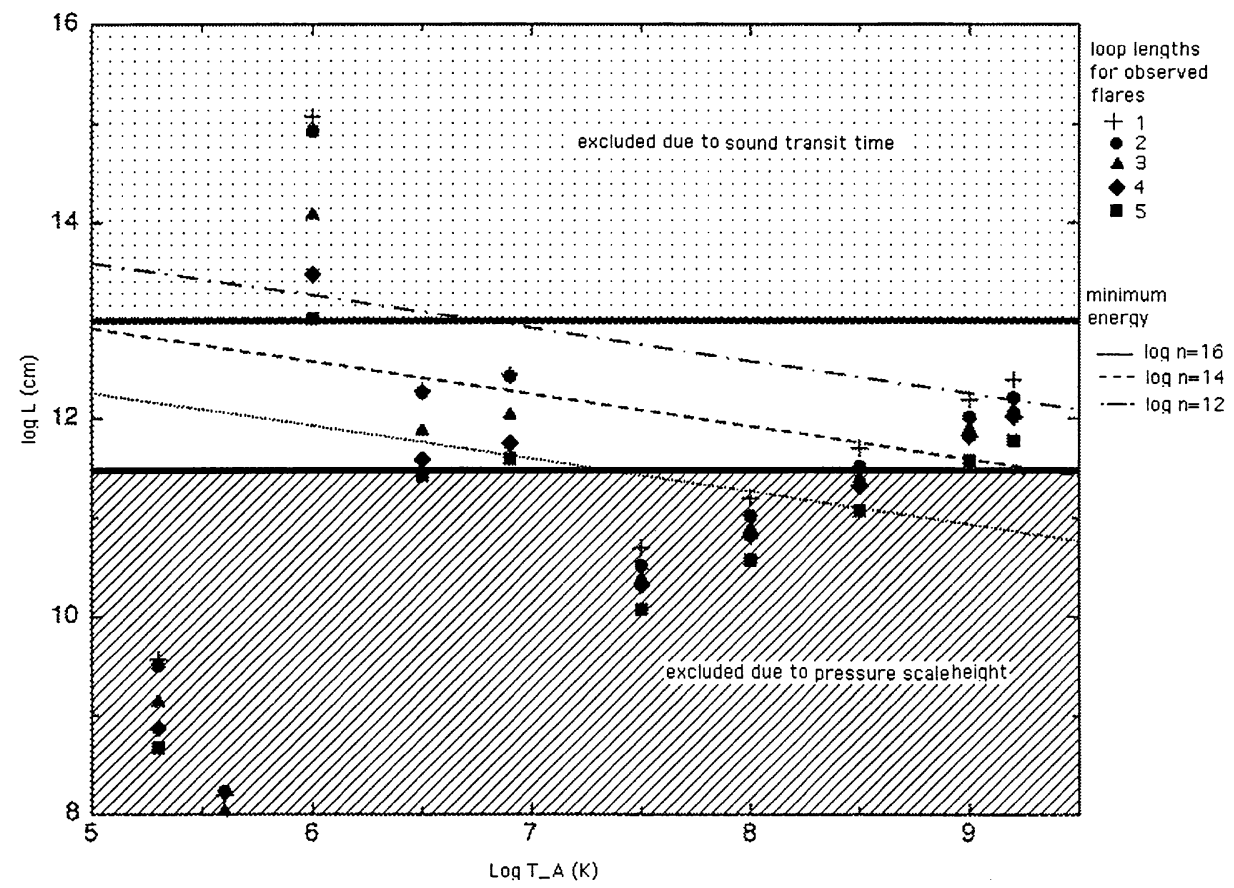

Figure 1. Predicted loop lengths of each flare for various temperatures are plotted against regions excluded by physical constraints.

height of the accretion disk; excluded regions are shown in Figure 1. Loop lengths fall within allowed regimes for temperatures above $10^{6.4} \mathrm{~K}$, which is consistent with accepted models of AGN. Thus, magnetic reconnection of loops is a strong possibility for the cause of variability in Seyfert galaxies.

\section{References}

DiMatteo, T. 1998, MNRAS, 299, L1

Fisher, G. H., \& Hawley, S. L. 1990, ApJ, 357, 243

Hawley, S. L., et al. 1995, ApJ, 453, 464

LeRoux, A. M. K., Longcope, D. W., Fisher, G. H., \& Tsuruta, S., in preparation Lin, R. P., \& Hudson, H. S. 1976, Solar Phys., 50, 153

Rosner, R., Tucker, W. H., \& Vaiana, G. S. 1978, ApJ, 220, 643 